\title{
Elastic wave velocity and electrical conductivity in a brine-saturated rock and microstructure of pores
}

\author{
Tohru Watanabe ${ }^{1 *} \mathbb{D}$, Miho Makimura ${ }^{1}$, Yohei Kaiwa' ${ }^{1}$, Guillaume Desbois ${ }^{2}$, Kenta Yoshida ${ }^{3}$ \\ and Katsuyoshi Michibayashi ${ }^{4}$
}

\begin{abstract}
Elastic wave velocity and electrical conductivity in a brine-saturated granitic rock were measured under confining pressures of up to $150 \mathrm{MPa}$ and microstructure of pores was examined with SEM on ion-milled surfaces to understand the pores that govern electrical conduction at high pressures. The closure of cracks under pressure causes the increase in velocity and decrease in conductivity. Conductivity decreases steeply below $10 \mathrm{MPa}$ and then gradually at higher pressures. Though cracks are mostly closed at the confining pressure of $150 \mathrm{MPa}$, brine must be still interconnected to show observed conductivity. SEM observation shows that some cracks have remarkable variation in aperture. The aperture varies from $100 \mathrm{~nm}$ to $\sim 3 \mu \mathrm{m}$ along a crack. FIB-SEM observation suggests that wide aperture parts are interconnected in a crack. Both wide and narrow aperture parts work parallel as conduction paths at low pressures. At high pressures, narrow aperture parts are closed but wide aperture parts are still open to maintain conduction paths. The closure of narrow aperture parts leads to a steep decrease in conductivity, since narrow aperture parts dominate cracks. There should be cracks in various sizes in the crust: from grain boundaries to large faults. A crack must have a variation in aperture, and wide aperture parts must govern the conduction paths at depths. A simple tube model was employed to estimate the fluid volume fraction. The fluid volume fraction of $10^{-4}-10^{-3}$ is estimated for the conductivity of $10^{-2} \mathrm{~S} / \mathrm{m}$. Conduction paths composed of wide aperture parts are consistent with observed moderate fluctuations $(<10 \%)$ in seismic velocity in the crust.
\end{abstract}

Keywords: Electrical conductivity, Seismic velocity, Fluid, Crack, Crust

\section{Introduction}

Aqueous fluids play important roles in crustal processes. They can decrease the frictional strength of faults (e.g., Sibson 2009) to cause seismic slips and reduce the flow strength of rocks through fluid-assisted mechanisms like pressure solution (e.g. Rutter 1983) to promote crustal deformation. The distribution of fluids is, thus, an important input for further understanding of crustal processes. Magnetotelluric surveys have revealed that the resistivity in the crust is much lower than that expected for dry rocks, suggesting the pervasive existence of fluids (e.g.,

\footnotetext{
*Correspondence: twatnabe@sus.u-toyama.ac.jp

1 Department of Earth Sciences, University of Toyama, Gofuku 3190,

Toyama, Japan

Full list of author information is available at the end of the article
}

Ogawa et al. 2001). The distribution of fluids, however, has not been quantitatively evaluated. A thorough understanding of electrical properties of fluid-bearing rocks is critical for the interpretation of observations.

Brace et al. (1965), in their pioneering work, showed that as the confining pressure was increased, the electrical resistivity of brine-saturated crystalline rocks increased at first sharply and then gradually. Brine is interconnected even at the confining pressure of $1 \mathrm{GPa}$. The sharp increase in resistivity at low pressure is caused by the closure of cracks with small aspect ratios. The electrical conduction at higher pressure must be maintained by pores with large aspect ratios. Similar observations have been reported on electrical resistivity (Lockner and Byerlee 1985) and permeability (Trimmer et al. 1980) of granitic rocks. 
Johnson and Manning (1986) tried the reproduction of the results of Brace et al. (1965). They calculated the effective resistivity of a network composed of cracks and pores, employing the effective medium theory (Kirkpatrick 1973). Cracks with small aspect ratios are easy to close at low pressures, while pores with large aspect ratios are open at high pressures. Their calculation suggested that connected cracks and connected pores work parallel as conduction paths at low pressure. At high pressure, the connected path of cracks is lost due to the closure of cracks and only connected pores give a conduction path.

Except for shallow depths, the electrical conduction in the crust should be governed by the connected path of pores with large aspect ratios. Though the nature of connected pores with large aspect ratios is, thus, a key for the interpretation of observed resistivity profiles, it has been poorly understood. A crack surface might be a distribution of asperities which progressively contact as the crack closes under pressure (Gangi 1978; Carlson and Gangi 1985). Based on the crack aperture distribution studied by Wong et al. (1989), Watanabe and Higuchi (2015) suggested that wide aperture parts in cracks work as conduction paths at high pressure. However, 3D structure of crack surfaces has been difficult to image. The distribution and connectivity of wide aperture parts have been poorly understood.

The aim of this study is to understand the nature of pores governing the electrical conduction at high pressure. We measured elastic wave velocities and electrical conductivity (the inverse of resistivity) simultaneously in a brine-saturated granitic rock under confining pressures and studied microstructure of pores with SEM observation on ion-milled surfaces. FIB-SEM images suggest the interconnection of wide aperture parts in a crack. The conduction paths in rock samples and in the crust will be discussed, and the fluid volume fraction in the crust will be estimated from observed resistivity.

\section{Experimental \\ Samples}

Aji granite (Aji, Kagawa prefecture, Japan) was used as a rock sample for its fine-grained $(100-500 \mu \mathrm{m})$ and equigranular texture. The rock sample consists of $52.8 \%$ plagioclase, 36.0\% quartz, 8.2\% biotite and 3.0\% K-feldspar (Watanabe and Higuchi 2015). Crystallographic orientation of mineral grains was analyzed with a scanning electron microscopy (SEM) electron backscattered diffraction (EBSD) system (HITACHI S-3400N Type II with HKL Channel 5) at Nagoya University. Orientations of crystal axes are shown in Fig. 1. The sharpness of a pole figure is characterized by the pfJ index, which takes a value of 1 for a random distribution (Michibayashi and
Mainprice 2004). Plagioclase, quartz and biotite show weak concentrations of crystal axes, while K-feldspar distinct concentration of axes (Maximum Density $=14.77$ 16.97). Since K-feldspar grains are sparsely distributed with the volume fraction of $3.0 \%$, the crystal-preferred orientation of K-feldspar can hardly contribute to the anisotropy in elasticity.

Samples have a cylindrical shape with dimensions of $26 \mathrm{~mm}$ in diameter and $30 \mathrm{~mm}$ in length. The apparent density is $2.631(5)-2.668(2) \mathrm{g} / \mathrm{cm}^{3}$ and the porosity is $1.5(7)-2.9(7) \%$ (Table 1$)$. The porosity in Table 1 was calculated with the density method (e.g., Guéguen and Palciauskas 1994). The porosity in AJG35 was measured also through the gas expansion method using a porosimeter (Shimadzu, Accupyc $1330 \mathrm{~V} 2.04 \mathrm{M}$ ) with helium gas at Earthquake Research Institute, the University of Tokyo. The gas expansion method measures the pore volume accessible from the sample outer surface. The porosity in AJG35 measured through the gas expansion method was $1.0(2) \%$. The larger porosity calculated with the density method suggests the existence of isolated pores.

The orthogonal directions were set for measurements of velocity and conductivity. The axial direction of a cylindrical sample was set to be the $Z$-axis direction, and two radial directions were the $X$ - and $Y$-axis directions. To determine the two radial directions, shear wave propagating in the axial direction was observed with changing the oscillation direction by $10^{\circ}$. The pulse transmission technique was employed using ultrasonic transducers (Panametrics, V153) with the central frequency of $1 \mathrm{MHz}$. A shear wave in an anisotropic medium splits into the fast and slow shear waves, which have mutually orthogonal oscillation directions (e.g., Mainprice 2007). When the oscillation direction is parallel to that of the fast shear wave, the amplitude of the fast shear wave is maximized. The oscillation direction of the fast shear wave was set to be the $X$-axis direction. The $Y$-axis direction is perpendicular to the $X$-axis direction. The polarization anisotropy in the $Z$-axis direction was quite weak $(\operatorname{AVs}(Z)<4 \%)$.

\section{Ultrasonic velocity measurements on a dry sample}

To study elastic properties of rock samples, ultrasonic velocities in a dry sample were measured. One compressional wave velocity and two shear wave velocities were measured in the $X-, Y$ - and $Z$-directions. In one propagation direction, two shear waves oscillate in mutually orthogonal directions. Measurements were conducted at ambient temperature. The confining pressures were increased up to $180 \mathrm{MPa}$ using a pressure vessel (Riken, PV-2M-S6F). The pressure medium was silicone oil (Shin-Etsu Chemical, KF-96-100cs). Ultrasonic velocities were measured through the pulse 

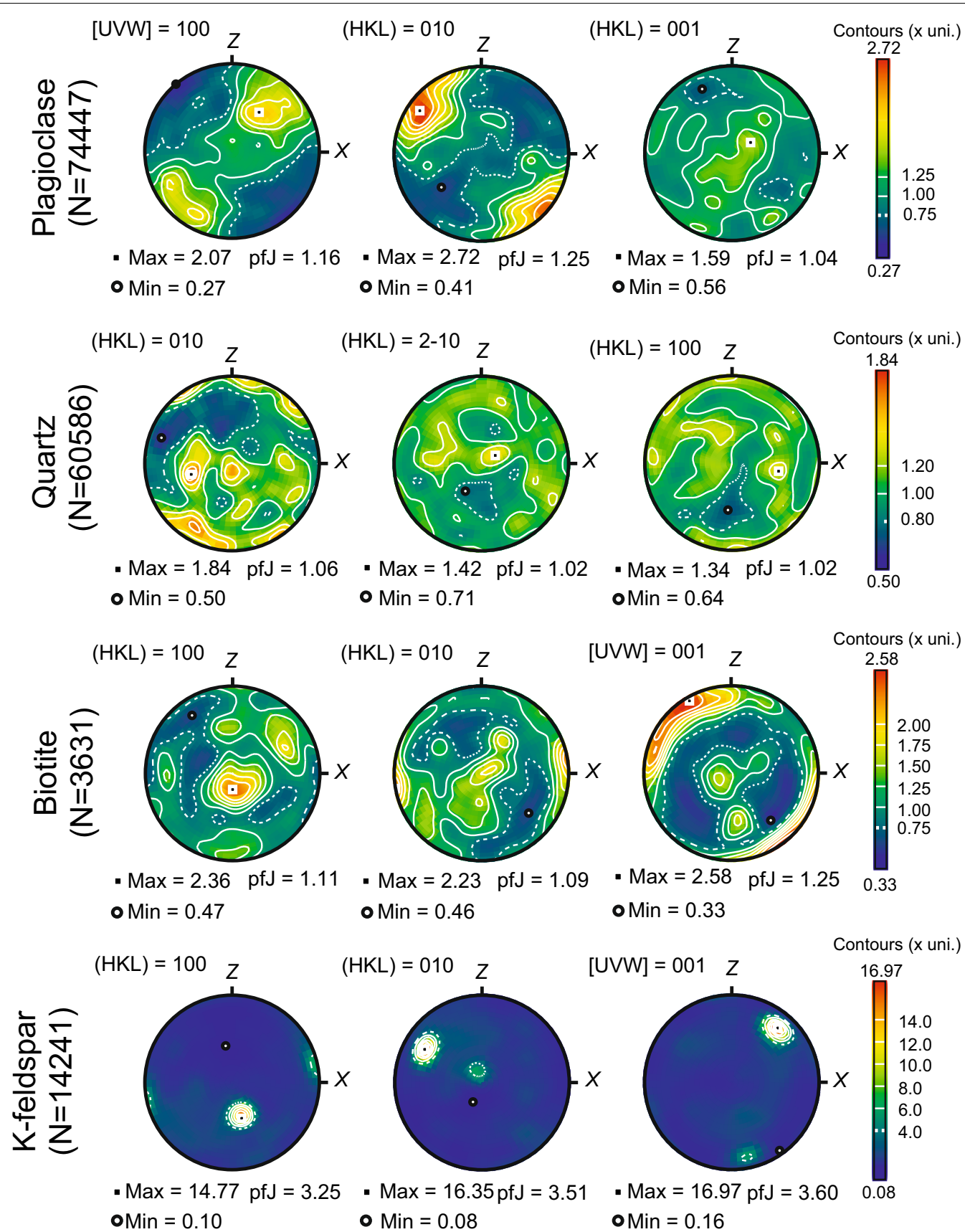

Fig. 1 Pole figures of crystallographic orientation of plagioclase, quartz, biotite and K-feldspar. $X$ - and Z-directions are the radial and axial directions of a cylindrical sample AJG02. Pole figures are equal-area lower-hemisphere projections with a Gaussian half-width angle of $8.5^{\circ}$. Contours represent the data points (multiples of uniform density). The maximum and minimum density values are shown. The pfJ index was proposed by Michibayashi and Mainprice (2004) to evaluate the fabric strength. The number of measured points is denoted by $N$

transmission technique using $\mathrm{Pb}(\mathrm{Zr}, \mathrm{Ti}) \mathrm{O}_{3}$ transducers (Fuji Ceramics, C-6) with the central frequency of $2 \mathrm{MHz}$. An RTV rubber (Shin-Etsu Chemical, KE-45) was used to cover a sample with glued transducers.
Pulse excitation and data acquisition were made by a function generator (Agilent Technologies, 33521A) and a digital oscilloscope (Agilent Technologies, DSO $\mathrm{X}-2022 \mathrm{~A})$, respectively. 
Table 1 List of samples

\begin{tabular}{llllll}
\hline Sample & Density $\left(\mathbf{g} / \mathbf{c m}^{\mathbf{3}}\right)$ & Porosity $(\%)$ & $\mathbf{V p}(\mathbf{Z})(\mathbf{k m} / \mathbf{s})$ & AVs(Z) (\%) & Measurements \\
\hline AJG02 & $2.668(2)$ & $1.9(7)$ & - & - & XCT, SEM \\
AJG35 & $2.631(5)$ & $2.9(7)$ & $5.07(2)$ & $3.4(4)$ & Velocity, conductivity (wet) \\
AJG50 & $2.646(5)$ & $2.4(7)$ & $5.06(1)$ & $1.3(2)$ & Velocity (dry) \\
AJG59 & $2.643(3)$ & $2.5(7)$ & $4.94(2)$ & $3.1(2)$ & Velocity, conductivity (wet)
\end{tabular}

The number inside brackets shows the error in the last digit

The porosity was calculated by the density method

$\operatorname{AVs}(Z)$ is calculated as $\operatorname{AVs}(Z)=\frac{\left|V_{s}(X Z)-V_{s}(Y Z)\right|}{\frac{1}{2}\left[\operatorname{Vs}(X Z)+V_{s}(Y Z)\right]} \times 100$

\section{Ultrasonic velocity and conductivity measurements on wet samples}

Ultrasonic velocities and electrical conductivity were simultaneously measured in brine-saturated rock samples at ambient temperature. The confining pressures were increased up to $150 \mathrm{MPa}$. The confining and porefluid pressures were independently controlled in a pressure vessel (Riken, PV-2M-S14). The pressure medium was silicone oil (Shin-Etsu Chemical, KF-96-100cs). A piston-cylinder system was used for the pore-fluid pressure transmission and the electrical isolation (Fig. 2a). One pump moves the piston to control the pore-fluid pressure. Watanabe and Higuchi (2014) examined the relationship between the pore-fluid and silicone oil pressures. The pore-fluid pressure in a sample can be controlled without a significant contribution of the friction between the piston and cylinder.
A cylindrical sample was evacuated, and then immersed in $0.1 \mathrm{~mol} / \mathrm{L} \mathrm{KCl}$ aqueous solution. It was assembled with stainless steel end-pieces in which stainless steel piston-cylinder systems are inserted (Fig. 2b). The aqueous pore fluid is isolated from silicone oil by two O-rings. The sample was covered with RTV rubber to prevent the infiltration of oil. The sample assembly was connected to the top block with two arms and a tie block (Fig. 2a). For electrical isolation between the pore fluid and the metal work of the pressure vessel, Teflon devices were placed between the sample assembly and the metal work (Fig. 2a). Along with the radial compression, a sample is axially compressed by the silicone oil between the top block and the upper end-piece and between the tie block and the lower end-piece, being under a hydrostatic compression. The right arm and the tie block work as a channel for silicone oil to move the lower piston (Fig. 2a).
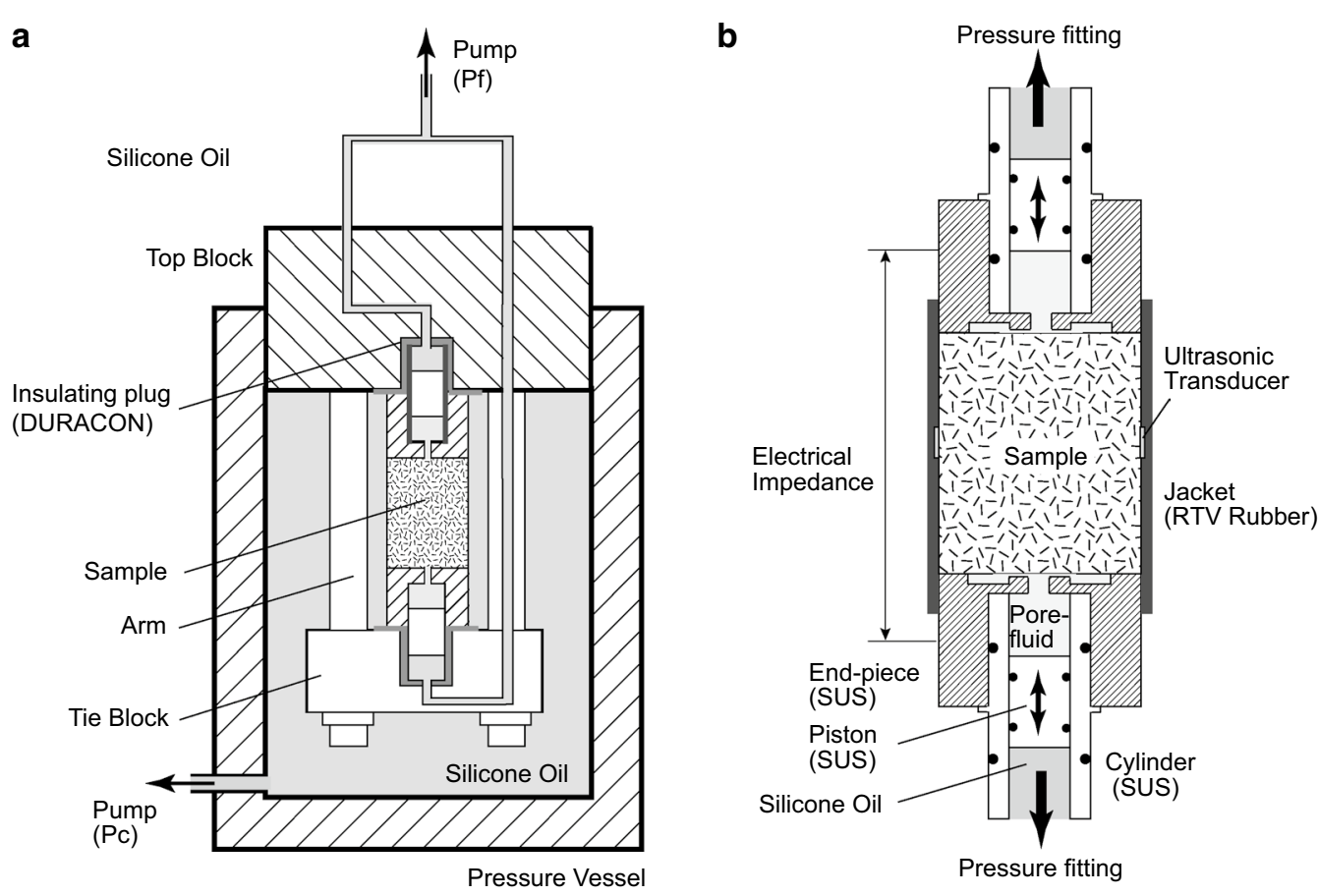

Fig. 2 Illustrations of a pressure vessel and $\mathbf{b}$ sample assembly 
The top block provides a channel for oil to move the upper piston.

Compressional wave velocity was measured in the $X$-axis direction, while shear wave velocity in the $Y$-axis direction. The shear wave oscillates parallel to the $Z$-axis direction. The pulse transmission technique was employed. The sample surface was coated with an epoxy resin for the electrical isolation between the pore fluid and transducers, and then $\mathrm{Pb}(\mathrm{Zr}, \mathrm{Ti}) \mathrm{O}_{3}$ transducers (Fuji Ceramics, C-6) with the central frequency of $2 \mathrm{MHz}$ were bonded to the surface. Pulse excitation and data acquisition were made by a function generator (Agilent Technologies, 33220A) and a digital oscilloscope (Agilent Technologies, DSO-X 2022A), respectively.

Electrical impedance was measured in the $Z$-axis direction with an LCR meter (NF, ZM2375). Stainless steel end-pieces were used as electrodes. The measurement frequency was from $1 \mathrm{~Hz}$ to $200 \mathrm{kHz}$. The measured resistance was converted to the conductivity by considering the length and cross-sectional area of a sample.

\section{Microstructural observation of pores}

Pores in a rock sample were examined using an X-ray computer-aided tomography (CT) system (V|tome|x L300, GE Sensing \& Inspection Technologies) at Tokyo Metropolitan Industrial Technology Research Institute, Jonan Branch. A cylindrical sample for X-ray CT ( $D=2 \mathrm{~mm}, L=6 \mathrm{~mm}$ ) was made from Sample AJG02 with ultrasonic machining at Institute for Planetary Materials, Okayama University.

Microstructure of cracks was studied using SEM. SEM observation was conducted on a surface polished with argon ion beam cross-sectioning (BIB, Broad Ion Beam) technique (e.g., Desbois et al. 2011). The Ar-BIB preparation technique produces a smooth and damage-free surface with relatively large area of up to $2 \mathrm{~mm}^{2}$, which is suitable for investigating microstructure of cracks. A sample surface was mechanically pre-polished to reduce roughness to ca. $20 \mu \mathrm{m}$; then, about $100 \mu \mathrm{m}$ of the outermost edge of the sample was polished by the BIB cross-section polisher (JEOL, SM09010) to remove prepolishing damage. The polishing was conducted using the ion beam of $6 \mathrm{kV}$ and $180 \mu \mathrm{A}$ for $8 \mathrm{~h}$. The BIB polished cross section was imaged using SEM (ZEISS, SUPRA 55). The BIB polishing and SEM imaging were conducted at RWTH Aachen University.

The 3D structure of a crack was investigated by serial sectioning using a dual-beam focused ion beam-SEM (FIB-SEM) system (Helios G4 UX, Thermo Fisher Scientific) at JAMSTEC, Yokosuka. A gallium ion source installed at an angle of $52^{\circ}$ was used to mill the sample. After careful determination of the target area, a $1-\mu \mathrm{m}$-thick platinum layer was deposited on the area of interest for the protection from the ion beam damage. Then, a large trench was milled around this area as a 'channel' for the removal of the sputtered material. An FIB serial sectioning was performed using the ion beam of $30 \mathrm{kV}$ and approximately $9.1 \mathrm{nA}$. SEM images were taken every $50 \mathrm{~nm}$ at an acceleration voltage of $15 \mathrm{kV}$ and a beam current of approximately $0.8 \mathrm{nA}$. 3D reconstruction of the serial-sectioned images was performed using Amira 6.3 (Thermo Fisher Scientific).

\section{Results}

\section{Pressure dependence of velocity in a dry sample}

Compressional and shear wave velocities in a dry sample (AJG50) are shown in Fig. 3 as a function of confining pressure. Measurements were made during the loading. The error in velocity is evaluated as

$$
\frac{\delta V}{V}=\sqrt{\left(\frac{\delta l}{l}\right)^{2}+\left(\frac{\delta t}{t}\right)^{2}},
$$

where $V, l$ and $t$ are the velocity, path length and travel time, respectively. The errors in these values are denoted by $\delta V, \delta l$ and $\delta t$. The relative error in velocity is $0.6-0.8 \%$ for compressional wave and $1-2 \%$ for shear wave. The error in velocity comes mainly from that in travel time. Both compressional and shear wave velocities increase with increasing confining pressure. The increase in velocity is larger for compressional waves $(\sim 1.3 \mathrm{~km} / \mathrm{s})$ than for shear waves $(\sim 0.6 \mathrm{~km} / \mathrm{s})$. The anisotropy in compressional wave velocity $(\mathrm{AVp})$ is $4.4(8) \%$ at atmospheric pressure and it decreases to $0.7(9) \%$ at $180 \mathrm{MPa}$. The polarization anisotropy of shear wave is not significant at $180 \mathrm{MPa}$. The pressure dependence of velocity is weakened at pressures higher than $100 \mathrm{MPa}$. The increase in velocity must thus be attributed to the closure of pores.

Elastic properties of the solid phase can be evaluated from velocities at $180 \mathrm{MPa}$, because the influence of pores on effective elastic properties is sufficiently weakened at high pressures. On the basis of weak anisotropy in velocity at $180 \mathrm{MPa}$, the isotropy in elasticity is assumed. Elastic constants of the solid phase are summarized in Table 2.

The closure pressure of pores depends on their shape. If we consider a spheroidal pore with aspect ratio $\alpha$ at atmospheric pressure, the closure pressure is given by

$$
p_{\text {closure }}=\frac{\pi E \alpha}{4\left(1-v^{2}\right)},
$$

where $E$ and $v$ are Young's modulus and Poisson's ratio of the solid phase (Walsh 1965). The aspect ratio $\alpha$ is defined by 

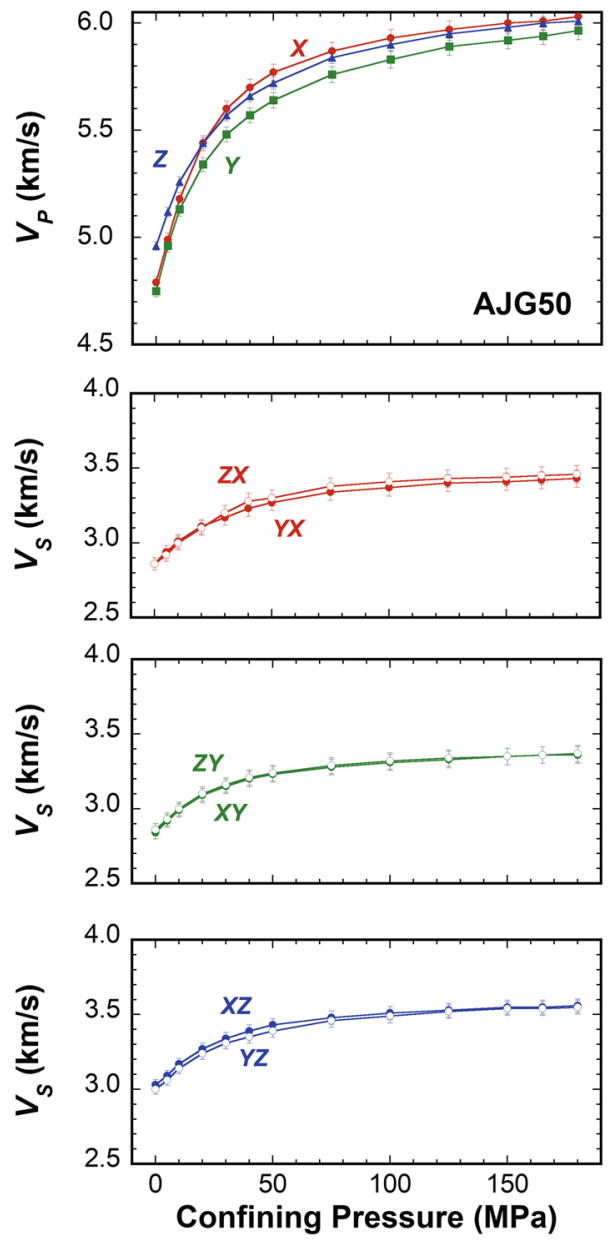

Fig. 3 Compressional and shear wave velocities in a dry rock sample AJG50 are shown as a function of confining pressure. The letter beside a compressional wave velocity represents the propagation direction. The first letter beside a shear wave velocity is the oscillation direction and the second letter the propagation direction. Z-direction is parallel to the axis of the cylindrical sample. $X$ - and $Y$-directions are perpendicular to $Z$-direction and mutually orthogonal

Table 2 Elastic properties of the solid phase

\begin{tabular}{ll}
\hline Properties & Estimated values \\
\hline Bulk modulus, $K_{S}$ & $53.5(9)(\mathrm{GPa})$ \\
Shear modulus, $G$ & $31.6(4)(\mathrm{GPa})$ \\
Young's modulus, $E$ & $79.3(9)(\mathrm{GPa})$ \\
Poisson's ratio, $V$ & $0.253(4)$ \\
\hline
\end{tabular}

$$
\alpha=\frac{c}{a}
$$

where $a$ and $c$ are the semi-major and minor axes of the spheroid. Pores with smaller aspect ratios, thus, close at lower pressures. The aspect ratio of pores closing at a given pressure can be estimated from Eq. (1) using elastic properties of the solid phase. The pores closed below $180 \mathrm{MPa}$ have aspect ratios smaller than $3 \times 10^{-3}$. Such oblate spheroidal pores are treated as cracks.

\section{Pressure dependence of velocity and conductivity in wet samples}

Velocities and conductivity in wet samples (AJG35, AJG59) are shown as a function of confining pressure in Fig. 4. For comparison, average velocities in the dry sample (AJG50) are also shown. The confining pressure was incrementally increased up to $150 \mathrm{MPa}$. The pore-fluid pressure was held at atmospheric pressure $(0.1 \mathrm{MPa})$. One confining pressure was held for around $50 \mathrm{~h}$ until velocities and conductivity became stationary values. Measurements were made during the loading. The error in velocity is evaluated as in a dry sample. The error in conductivity is evaluated as
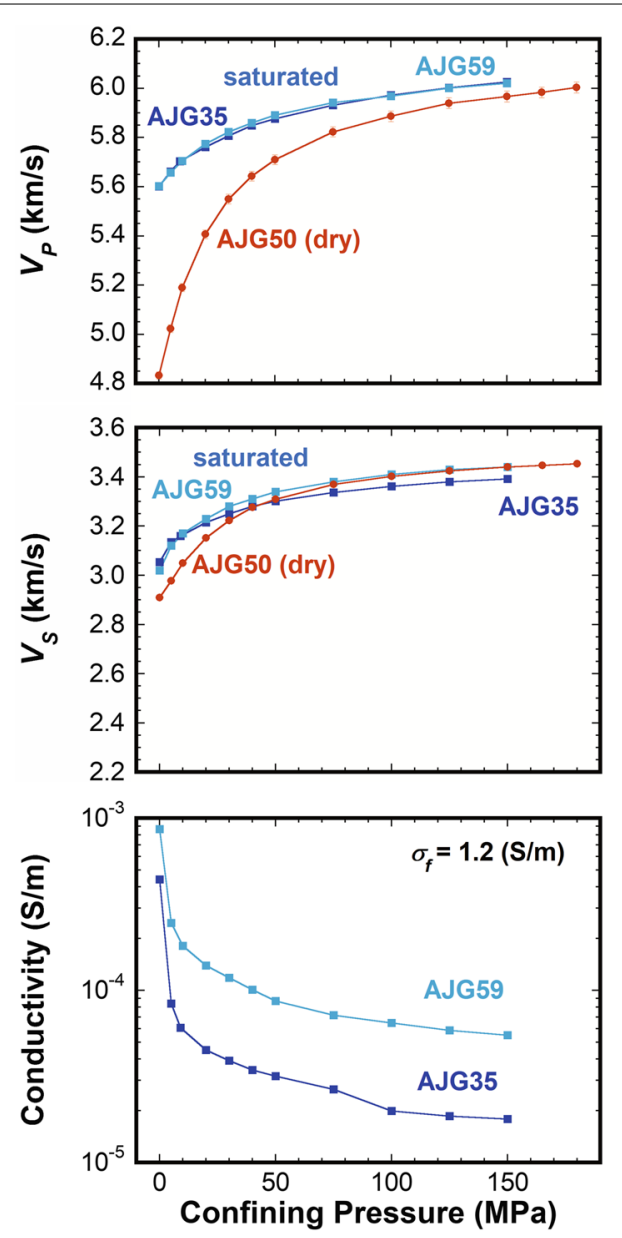

Fig. 4 Velocities and electrical conductivity in brine-saturated samples are shown as a function of confining pressure. The pore-fluid pressure was kept at atmospheric pressure $(0.1 \mathrm{MPa})$. For comparison, average velocities in dry sample AJG50 are also shown 


$$
\frac{\delta \sigma}{\sigma}=\sqrt{\left(\frac{\delta R}{R}\right)^{2}+\left(\frac{\delta l}{l}\right)^{2}+4\left(\frac{\delta r}{r}\right)^{2}},
$$

where $\sigma, R, l$ and $r$ are conductivity, resistance, length and radius of the sample, respectively. The errors in these values are denoted by $\delta \sigma, \delta R, \delta l$ and $\delta r$. The relative error in conductivity is $5-10 \%$. The error in conductivity comes mainly from that in resistance.

Velocities increase and conductivity decreases with increasing confining pressure. Velocities in wet samples are higher than those in the dry sample. Compressional and shear wave velocities increase by $0.4 \mathrm{~km} / \mathrm{s}(7 \%)$ and $0.3 \mathrm{~km} / \mathrm{s}(10 \%)$ as the confining pressure was increased from 0.1 to $150 \mathrm{MPa}$. Larger increase in velocity is observed at confining pressures lower than $50 \mathrm{MPa}$. The increase in velocities must be attributed to the closure of pores under pressure, because the difference between velocities in wet and dry samples decreases with increasing confining pressure.

Conductivity shows a large drop with the increase in confining pressure. Conductivity decreases by about one order of magnitude as the confining pressure was increased from 0.1 to $10 \mathrm{MPa}$, and shows gradual decrease at higher pressures. The decrease rate of conductivity is reduced with increasing confining pressure. Similar changes in conductivity have been observed in previous studies (Brace et al. 1965; Lockner and Byerlee 1985; Watanabe and Higuchi 2015). The observed conductivity clearly demonstrates the connectivity of brine in samples. The conductivity drop must also be attributed to the closure of pores under pressure.

\section{Microstructure of pores}

$\mathrm{X}$-ray CT images were taken over a volume of $2 \mathrm{~mm}$ in diameter and $3 \mathrm{~mm}$ in length. Since the dimension of one voxel is $3 \mu \mathrm{m}$, narrow cracks cannot be clearly imaged. Though the spatial resolution is not enough for imaging microstructure of pores, X-ray CT images are useful for examining the distribution of pores and their connectivity. Thin cracks and round pores are imaged by X-ray CT (Fig. 5a, b). Cracks are categorized as grain boundary crack (open grain boundary) or intragrain crack. Open grain boundaries are pervasive in the rock sample. Plagioclase grains have many intragrain cracks (Fig. 5b). Round pores, which must be traces of fluids, are isolated in grains (Fig. 5a). These round pores could explain the discrepancy between porosities measured by the density and gas expansion methods.

BIB-SEM images clearly show microstructure of pores, though the polished area is limited up to $2 \mathrm{~mm}^{2}$. Some cracks show remarkable variation in aperture (Fig. 5c). The aperture varies from $\sim 100 \mathrm{~nm}$ to $\sim 3 \mu \mathrm{m}$ along a crack. Narrow aperture parts will be closed under a low
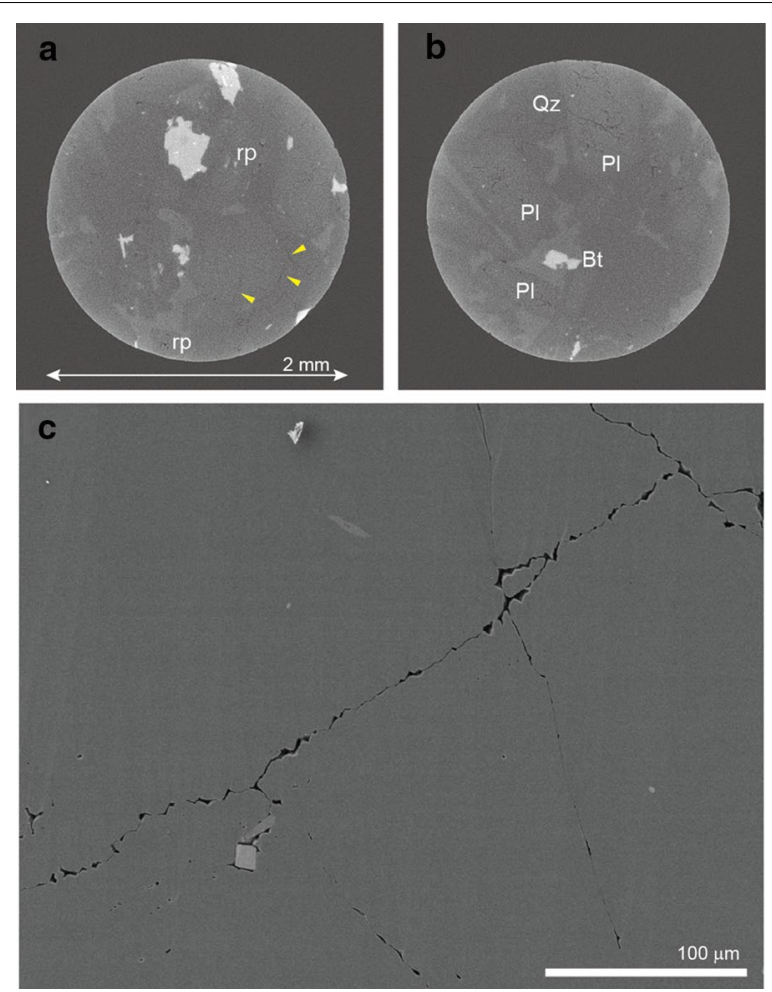

Fig. 5 a, $\mathbf{b}$ Radial cross-sectional images of X-ray CT of a cylindrical sample ( $D=2 \mathrm{~mm}, L=6 \mathrm{~mm}$ ). Cross sections are parallel to $Z X$-plane of a cylindrical sample AJG02. Lots of cracks (arrows) and round pores (rp) are imaged, and there many intragrain cracks in plagioclase grains. c An SEM image of ion-milled surface of AJG02

pressure, while wide aperture parts will remain opened even under high pressures because of their high aspect ratios. If wide aperture parts are interconnected in a crack, they can form an interconnected network and maintain the conduction path at high pressures.

FIB-SEM images provide us 3D structure of a crack, though the milled area is further limited to $20 \times 40 \mu \mathrm{m}^{2}$ (Fig. 6). The aperture varies along the crack and with depth. Wide aperture parts can be seen to the maximum depth of $30 \mu \mathrm{m}$ and look interconnected within the observed area.

\section{Discussion}

\section{Closure of cracks under pressure}

The change in velocity and conductivity must be caused by the closure of cracks under pressure. The quantity of crack is characterized by the crack density parameter defined as

$$
\varepsilon=\frac{1}{V} \sum_{i} a_{i}^{3}
$$



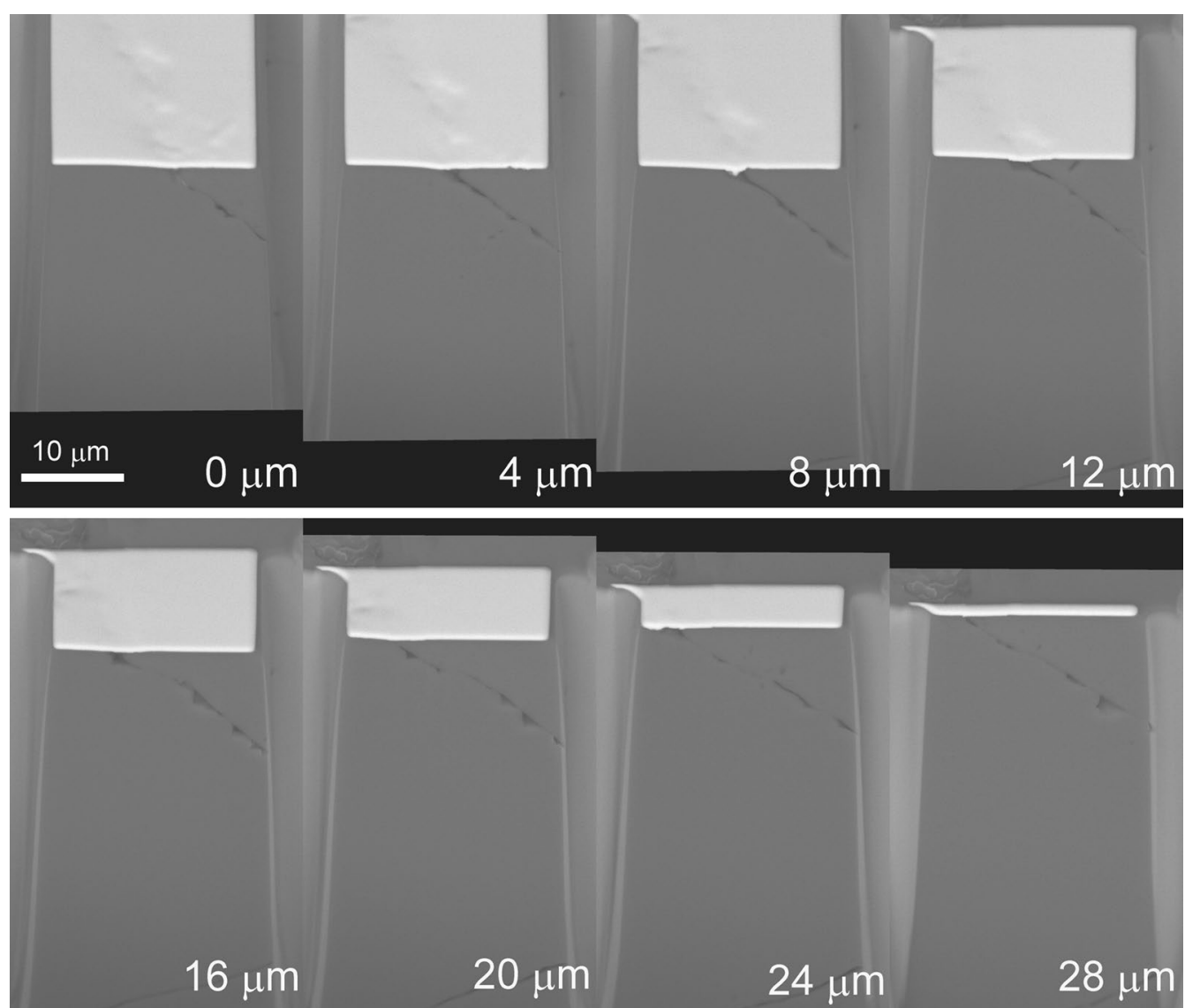

Fig. 6 SEM images of progressive slices of a crack in a dry sample AJG02. A white area in the upper part is a platinum layer for the protection from the ion beam damage

where $a_{i}$ is the radius of the $i$ th crack and the sum is conducted over all cracks in a volume $V$ (O'Connell and Budiansky 1974). The crack density parameter is proportional to the volume fraction of stress-released space around cracks. Effective elastic properties in a dry sample are insensitive to the aspect ratio of cracks, since the ratio of bulk moduli of fluid and solid phases $K_{\mathrm{f}} / K_{\mathrm{s}}$ is negligibly small compared with the aspect ratio of cracks (O'Connell and Budiansky 1974). Compressional- and shear-wave velocities in a dry sample are, thus, calculated as a function of the crack density parameter using equations in O'Connell and Budiansky (1974). Effective elastic properties in a wet sample depend also on the aspect ratio of cracks (e.g., O'Connell and Budiansky 1974). Velocities in a wet sample are calculated as a function of the crack density parameter and aspect ratio using equations in Schmeling (1986), which are a compilation of previous studies including O'Connell and Budiansky (1974). The crack density parameter and aspect ratio are determined to minimize

$$
S=\left(\frac{V_{\mathrm{P}}^{\text {calc }}-V_{\mathrm{P}}^{\text {meas }}}{V_{\mathrm{P}}^{\text {meas }}}\right)^{2}+\left(\frac{V_{\mathrm{S}}^{\text {calc }}-V_{\mathrm{S}}^{\text {meas }}}{V_{\mathrm{S}}^{\text {meas }}}\right)^{2} .
$$

The crack density parameter and aspect ratio are shown in Fig. 7 as a function of confining pressure. The aspect ratio is varied from $1 \times 10^{-5}$ to $1 \times 10^{-2}$. Estimation errors of the crack density parameter and aspect ratio are evaluated from the condition

$$
S \leq\left(\frac{\delta V_{\mathrm{P}}^{\text {meas }}}{V_{\mathrm{P}}^{\text {meas }}}\right)^{2}+\left(\frac{\delta V_{\mathrm{S}}^{\text {meas }}}{V_{\mathrm{S}}^{\text {meas }}}\right)^{2},
$$

where $\delta V_{\mathrm{P}}^{\text {meas }}$ and $\delta V_{\mathrm{S}}^{\text {meas }}$ are the errors of measured velocities. The crack density parameter is well determined, while the aspect ratio is poorly constrained. For a given crack density parameter, the influence of cracks on elasticity decreases with decreasing aspect ratio. For small aspect ratios $\left(<10^{-3}\right)$, the velocity difference becomes quite small for different aspect ratios. The 

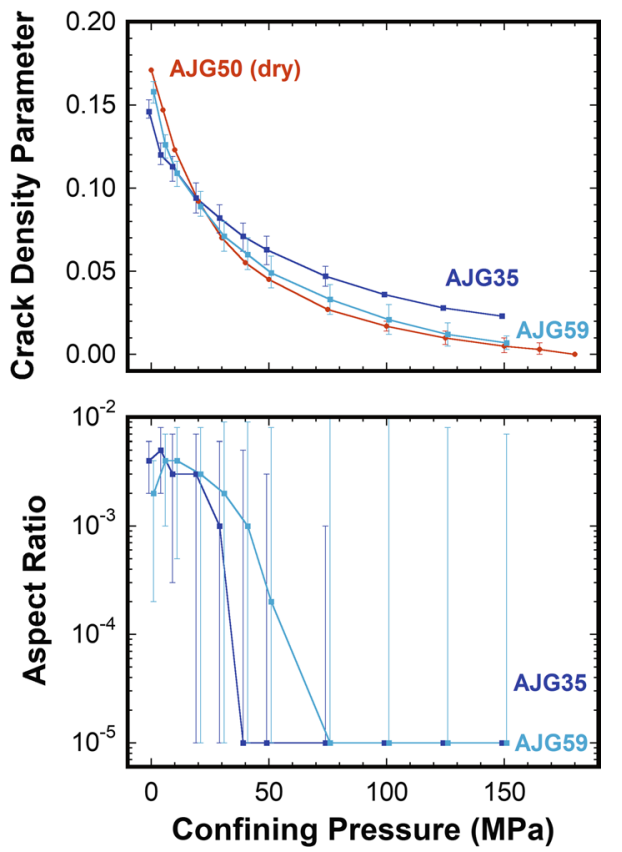

Fig. 7 Estimated crack density parameter and aspect ratio as a function of confining pressure. The crack density parameter is relatively well constrained, while aspect ratio poorly constrained, especially for aspect ratio less than $3 \times 10^{-3}$

aspect ratio larger than $4 \times 10^{-3}$ is, thus, relatively well constrained. The crack density parameter decreases with increasing confining pressure. It steeply drops at low pressures $(<20 \mathrm{MPa})$ and the decreasing rate is reduced with increasing pressure.

The decrease in crack density parameter reflects the closure of cracks under pressure. If cracks are ideal oblate spheroids, they are closed, respectively, at the closure pressures determined by their aspect ratios at atmospheric pressure. The steep decrease in crack density parameter at low pressure implies that cracks with small aspect ratios dominate the crack density parameter at atmospheric pressure. More cracks have smaller aspect ratios, or cracks with smaller aspect ratios have larger radii. Cracks in our samples, however, have complex shapes (Fig. 5c) to be segmented under pressure. Narrow aperture parts are further segmented or closed at low pressures, while wide aperture parts remain open to high pressures. The steep decrease in crack density parameter is caused by the crack segmentation and the dominance of narrow aperture parts, which can be seen in microstructures. Wong et al. (1989) measured the crack surface area as a function of aperture in rock

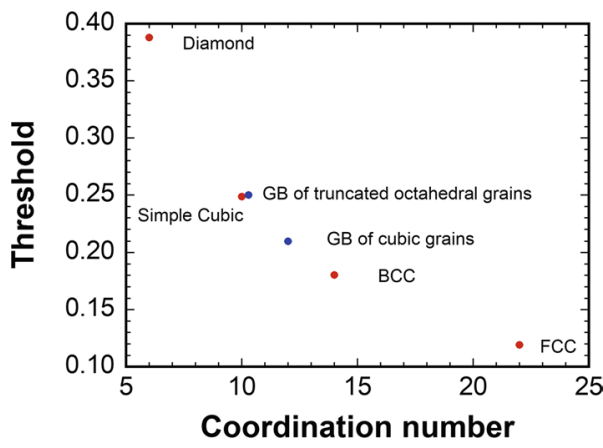

Fig. 8 Threshold for bond percolation as a function of coordination number. Threshold values for diamond, simple cubic, body-centered cubic (BCC) and face-centered cubic (FCC) lattices are 0.388, 0.2488, 0.1803 and 0.119 , respectively (Stauffer and Aharony 1994)

samples and showed that the crack surface area steeply decreases its aperture.

\section{Nature of conduction path}

Cracks in rock samples form an interconnected network at atmospheric pressure. Measured conductivity in wet samples requires an interconnected path of brine, which fills cracks. The formation of an interconnected network of cracks is treated as a problem of percolation (e.g., Guéguen and Palciauskas 1994). When the crack density is higher than a critical value, an interconnected network of cracks is formed. The critical value is called percolation threshold and depends on the configuration of network. The percolation threshold is analytically evaluated if cracks form a Bethe lattice (Guéguen and Dienes 1989).

The percolation of open grain boundary (grain boundary crack) is considered, since X-ray CT images shows that open grain boundaries are pervasive in a rock sample (Fig. 5a). When we consider 3D arrays of cubic and octahedral grains, the percolation threshold is $21 \%$ and $24 \%$, respectively (Appendix). The threshold for the bond percolation tends to decrease with increasing coordination number of a network (Fig. 8). To study the coordination number of grain boundary in our rock sample, a 3D image of grain boundaries was constructed from X-ray $\mathrm{CT}$ images and the coordination number was counted on 19 grain boundaries. The observed coordination number is from 7 to 11 , and the average coordination number is 8.7. Considering the relationship between percolation threshold and coordination number (Fig. 8), more than $25 \%$ of grain boundaries must be opened to form an interconnected network.

Wide aperture parts must govern conductivity at high pressures. FIB-SEM images suggest that wide aperture parts are interconnected in a crack. Though cracks are mostly closed at high pressures, wide aperture parts 
can maintain their interconnection within a crack. Wide aperture parts must form an interconnected network in a rock sample to show observed conductivity at high pressures. Cracks without wide aperture parts are completely closed under pressure. The fraction of crack with connected wide aperture parts must be larger than the percolation threshold to maintain the connectivity of brine at high pressures.

We evaluate the quantity of wide aperture parts required to show observed conductivity by employing a simple geometrical model. Wide aperture parts are treated as square tubes with the cross-sectional area of $a^{2}$. Brine-filled square tubes are embedded in an insulating cube with the edge length of $L$ (Fig. 9a). When the number of tubes is $n$, the effective conductivity is given by

$$
\sigma_{\mathrm{eff}}=\sigma_{\mathrm{f}} n\left(\frac{a}{L}\right)^{2},
$$

where $\sigma_{\mathrm{f}}$ is the conductivity of brine. If the dimension of tube, $a$ is given, the number of tubes in a given area can be evaluated from the conductivity at high pressure. The dimension of tube is set to be $1 \mu \mathrm{m}$ from the SEM observation. The effective and brine conductivities are of the order of $10^{-5}$ and $1 \mathrm{~S} / \mathrm{m}$, respectively. When we set $L$ to be $1 \mathrm{~mm}, n$ is of the order of 10 per $1 \mathrm{~mm}^{2}$. The number of wide aperture parts is estimated to be 120 in $1 \mathrm{~mm}^{2}$ from the examination of an SEM image of $0.5 \mathrm{~mm}^{2}$. Around $10 \%$ of wide aperture parts must be effectively involved in the interconnected network. The rest of wide aperture parts are isolated or dead-ended.

The closure of narrow aperture parts causes the steep decrease in conductivity. At low pressure, the connected paths of narrow aperture parts work parallel with those of wide aperture parts. As the pressure is increased, narrow aperture parts are progressively closed and the connected paths of narrow aperture parts are lost. We here evaluate the dimension of connected path of narrow aperture
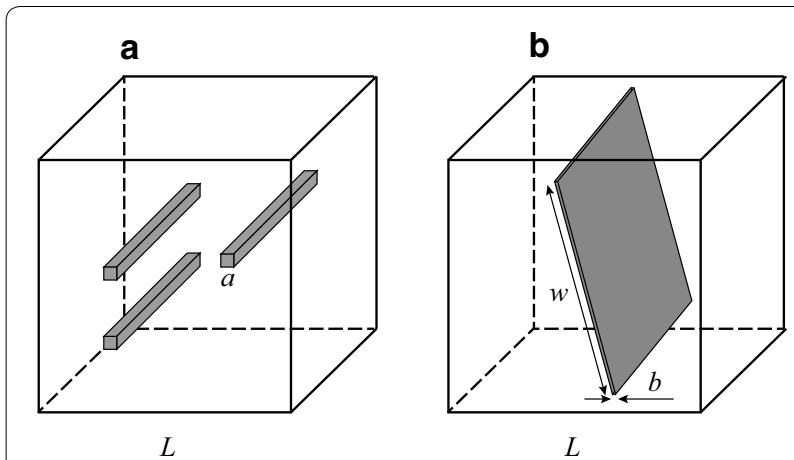

Fig. 9 Geometrical models of wide aperture parts (a) and narrow aperture parts (b) parts. Narrow aperture parts are treated as a film with the thickness $b$ and width $w$ and embedded in an insulating cube with the edge length of $L$ (Fig. 9b). The effective conductivity is given by

$$
\sigma_{\mathrm{eff}}=\sigma_{\mathrm{f}} \frac{b w}{L^{2}} .
$$

The thickness $b$ is set to be $0.1 \mu \mathrm{m}$ from the SEM observation. The effective conductivity at atmospheric pressure is $(4-8) \times 10^{-4} \mathrm{~S} / \mathrm{m}$. When we set $L$ to be $1 \mathrm{~mm}$, $w$ is $4-8 \mathrm{~mm}$. This value is supported by the stereological analysis of microstructures. The length of features per unit area $L_{\mathrm{A}}$ is related to the average number of interceptions of features per unit length of the test lines $N_{\mathrm{L}}$ as

$$
L_{\mathrm{A}}=\frac{\pi}{2} N_{\mathrm{L}}
$$

(Underwood 1970). Examination of narrow aperture parts on the SEM image (Fig. 5c) leads to $13.5 \mathrm{~mm} / \mathrm{mm}^{2}$. $30-60 \%$ of narrow aperture parts must effectively interconnected to show observed conductivity at atmospheric pressure.

\section{Conduction path in the crust}

In the crust, there should be cracks with various sizes: from grain boundaries to large faults. A crack must have a variation in the aperture. Narrow aperture parts are closed under pressure, while wide aperture parts are open to govern electrical conductivity at depths. Observed variation in conductivity must reflect the variation in quantity of wide aperture parts. More cracked regions might have more wide aperture parts.

The amount of fluid in the crust can be estimated by employing a simple geometrical model. We treat connected wide aperture parts as tubes. The effective conductivity for randomly oriented tubes is given by

$$
\sigma_{\mathrm{eff}}=\frac{1}{3} \phi \sigma_{\mathrm{f}}+(1-\phi) \sigma_{\mathrm{s}} \approx \frac{1}{3} \phi \sigma_{\mathrm{f}}
$$

where $\phi$ and $\sigma_{\mathrm{s}}$ are the fluid volume fraction and the conductivity of solid phase (Grant and West 1965). The fluid conductivity in the crust is estimated to be $10-100 \mathrm{~S} / \mathrm{m}$ (Nesbit 1993). The fluid volume fraction of $10^{-4}-10^{-3}$ is thus expected for the effective conductivity of $10^{-2} \mathrm{~S} / \mathrm{m}$. This fluid fraction is a minimum estimation, because there should be some fluid isolated from a connected network. If $50 \%$ of fluid is isolated, the estimation of fluid volume fraction will double. For a given fluid volume fraction, fluid-filled tubes give much smaller decrease in seismic velocity than fluid-filled cracks (e.g., Takei 2002). Conduction paths composed of wide aperture parts are consistent with observed moderate fluctuations $(<10 \%)$ in seismic velocity in the crust (e.g., Matsubara et al. 2004). 


\section{Conclusions}

To understand the pores that govern electrical conduction at high pressures, simultaneous measurements of elastic wave velocity and electrical conductivity were conducted on a brine-saturated granitic rock under confining pressures of up to $150 \mathrm{MPa}$ and microstructure of pores was examined with BIB-SEM and FIB-SEM. Cracks are closed under confining pressure to increase velocity and decrease conductivity. Conductivity decreases steeply below $10 \mathrm{MPa}$ and then gradually at higher pressures. Though cracks are mostly closed at the confining pressure of $150 \mathrm{MPa}$, brine is still interconnected to observed conductivity.

BIB-SEM observation shows that some cracks have remarkable variation in aperture. The aperture varies from $\sim 100 \mathrm{~nm}$ to $\sim 3 \mu \mathrm{m}$ along a crack. FIB-SEM observation suggests that wide aperture parts are interconnected in a crack. Both wide and narrow aperture parts work parallel as conduction paths at low pressures. At high pressures, narrow aperture parts are closed but wide aperture parts are still open to maintain conduction paths. The closure of narrow aperture parts leads to a steep decrease in conductivity, since narrow aperture parts dominate cracks.

There should be cracks in various sizes in the crust: from grain boundaries to large faults. A crack must have a variation in the aperture and wide aperture parts must govern the conduction paths at depths. A simple tube model was employed to estimate the fluid volume fraction. The fluid volume fraction of $10^{-4}-10^{-3}$ is estimated for the conductivity of $10^{-2} \mathrm{~S} / \mathrm{m}$. Conduction paths composed of wide aperture parts are consistent with observed moderate fluctuation $(<10 \%)$ in seismic velocity in the crust.

\section{Abbreviations}

SEM: scanning electron microscopy; EBSD: electron backscattered diffraction; BIB: broad ion beam; FIB: focused ion beam; CT: computer-aided tomography.

\section{Acknowledgements \\ Yves Guéguen and an anonymous reviewer are thanked for their careful reading and constructive comments. We thank T. Takezawa for conducting $X$-ray CT dat Tokyo Metropolitan Industrial Technology Research Institute. We are grateful to $\mathrm{A}$. Yoneda for ultrasonic machining of samples for X-ray CT, F. Maeno for his help in measuring density by the gas expansion method, and Riken-Seiki for designing and building our high-pressure apparatuses.}

\section{Authors' contributions}

TW proposed the topic and designed the study. MM and TW carried out the experimental study. YK and TW conducted numerical experiments of percolation. GD and KY conducted BIB-SEM and FIB-SEM observations, respectively. KM studied the orientation of mineral grains with SEM-EBSD. All authors read and approved the final manuscript.

\section{Funding}

This work was supported by the Grant-in-Aid for Scientific Research on Innovative Areas (KAKENHI No.2608) from the Ministry of Education, Culture,
Sports, Science and Technology (MEXT), Japan. It was also supported by JSPS KAKENHI 17 K05628.

Availability of data and materials

All data used in the present paper are available from the authors upon request.

Ethics approval and consent to participate

Not applicable.

Consent for publication

Not applicable.

\section{Competing interests}

The authors declare that they have no conflict of interest.

\section{Author details}

'Department of Earth Sciences, University of Toyama, Gofuku 3190, Toyama, Japan. ${ }^{2}$ Structural Geology, Tectonics and Geothermics, RWTH Aachen University, Lochnerstrasse 4-20, Aachen, Germany. ${ }^{3}$ Research and Development Center for Ocean Drilling Science, Japan Agency for Marine-earth Science and Technology, Natsushima-cho 2-15, Yokosuka, Japan. ${ }^{4}$ Department of Earth and Planetary Sciences, Nagoya University, Furo-cho, Chikusa-ku, Nagoya, Japan.

\section{Appendix: Percolation of open grain boundaries}

For simplicity, the three-dimensional arrays of cubic and truncated-octahedral grains are considered. Both cube and truncated octahedron are space-filling polyhedrons. Grains are assumed to have an identical shape, and open grain boundaries are randomly distributed. The length and size of clusters are measured for various fractions of open grain boundary

Numerical experiments were conducted on an array of $50 \times 50 \times 50$ grains. The normalized length of the largest cluster is shown in Fig. 10a as a function of the fraction of open grain boundary. The length is defined as the largest dimension in three orthogonal directions and normalized by the dimension of the array (50). Experiments were conducted 10 times for a fraction of open grain boundary, and the normalized length is averaged. If more than $21 \%$ of grain boundaries are open, an interconnected path is formed over the cubic array. The fraction of $21 \%$ is thus the percolation threshold for open grain boundaries in the cubic array. The percolation threshold is $24 \%$ for the array of truncated-octahedral grains.

The connectivity also shows a steep increase around the threshold (Fig. 10b). The size of a cluster is the number of open grain boundaries in a cluster. The connectivity is defined by the ratio of the largest cluster size to the total number of open grain boundaries. It is the probability for an open grain boundary to belong to the largest cluster. The connectivity is $0.6-0.8$ at the threshold, and it increases to 1.0 as the open grain boundary fraction increases to $40 \%$. 

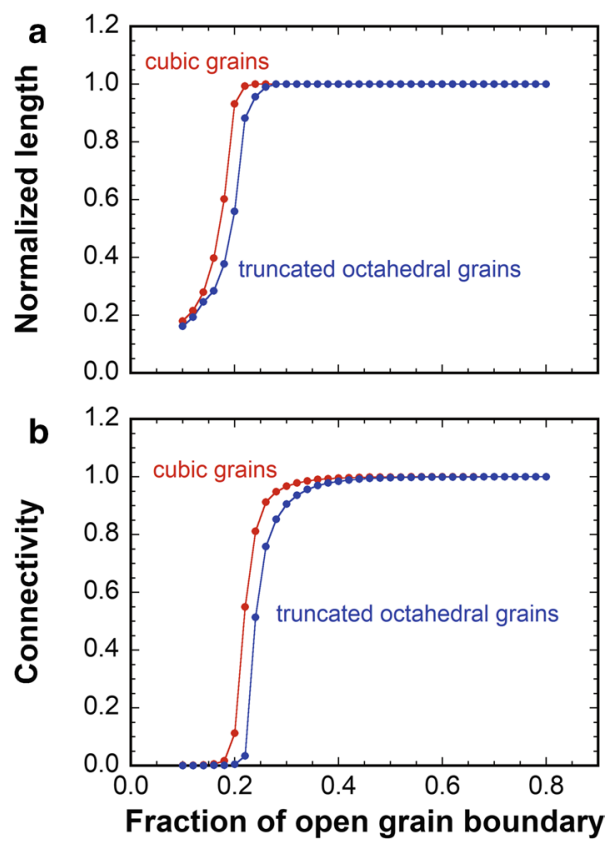

Fig. 10 Normalized length of the largest cluster and connectivity for arrays of cubic and truncated-octahedral grains. a Normalized length of the largest cluster as a function of the fraction of open grain boundary. The length is normalized by the dimension of the array (50) and averaged for 10 numerical experiments. b Connectivity as a function of the fraction of open grain boundary. The connectivity is defined by the ratio of the largest cluster size to the total number of open grain boundaries. The size of a cluster is the number of open grain boundaries in a cluster. The connectivity is also averaged for 10 numerical experiments

Received: 29 July 2019 Accepted: 21 November 2019

Published online: 29 November 2019

\section{References}

Brace WF, Orange AS, Madden TR (1965) The effect of pressure on the electrical resistivity of water-saturated crystalline rocks. J Geophys Res 10:5669-5678

Carlson RL, Gangi AF (1985) Effects of cracks on the pressure dependence of P wave velocities in crystalline rocks. J Geophys Res 90:8675-8684

Desbois G, Urai UL, Kukla PA, Konstanty J, Baerle C (2011) High-resolution 3D fabric and porosity model in a tight gas sandstone reservoir: a new approach to investigate microstructures from $\mathrm{mm}$ - to nm-scale combining argon beam cross-sectioning and SEM imaging. J Petrol Sci Eng 78:243-257

Gangi AF (1978) Variation of whole and fractured porous rock permeability with confining pressure. Int J Rock Mech Min Sci 15:249-257

Grant FS, West GF (1965) Interpretation theory in applied geophysics. McGrawHill, New York

Guéguen Y, Dienes J (1989) Transport properties of rocks from statistics and percolation. Math Geol 21:1-13

Guéguen Y, Palciauskas V (1994) Introduction to the physics of rocks. Princeton University Press, Princeton

Johnson DL, Manning H (1986) Theory of pressure dependent resistivity in crystalline rocks. J Geophys Res 91:11611-11617

Kirkpatrick S (1973) Percolation and conduction. Rev Mod Phys 45:574-588

Lockner DA, Byerlee JD (1985) Complex resistivity measurements of confined rock. J Geophys Res 90:7837-7847

Mainprice D (2007) Seismic anisotropy of the deep earth from a mineral and rock physics perspective. In: Schubert G (ed) Treatise on geophysics, vol 2. Elsevier, Amsterdam, pp 437-492

Matsubara M, Hirata N, Sato H, Sakai S (2004) Lower crustal fluid distribution in the northeastern Japan arc revealed by high-resolution 3D seismic tomography. Tectonophys 388:33-45

Michibayashi K, Mainprice D (2004) The role of pre-existing mechanical anisotropy on shear zone development within oceanic mantle lithosphere: an example from the Oman ophiolite. J Petrol 45:405-414

Nesbit BE (1993) Electrical resistivities of crustal fluids. J Geophys Res 98:4301-4310

O'Connell RJ, Budiansky B (1974) Seismic velocity in dry and saturated cracked solids. J Geophys Res 79:5412-5426

Ogawa Y, Mishina M, Goto T, Satoh H, Oshiman N, Kasaya T, Takahashi Y, Nishitani T, Sakanaka S, Uyeshima M, Takahashi Y, Honkura Y, Matsushima M (2001) Magnetotelluric imaging of fluids in intraplate earthquake zones, NE Japan back arc. Geophys Res Lett 28:3741-3744

Rutter EH (1983) Pressure solution in nature, theory and experiment. J Geol Soc Lond 140:725-740

Schmeling H (1986) Numerical models on the influence of partial melt on elastic, anelastic and electric properties of rocks. Part l: elasticity and anelasticity. Phys Earth Planet Inter 41:43-57

Sibson R (2009) Rupturing in overpressured crust during compressional inversion-the case from NE Hoshu, Japan. Tectonophysics 473:404-416

Stauffer D, Aharony A (1994) Introduction to percolation theory. CRC Press, Boca Raton

Takei Y (2002) Effect of pore geometry on Vp/Vs: from equilibrium geometry to crack. J Geophys Res. https://doi.org/10.1029/2001JB000522

Trimmer D, Bonner B, Heard HC, Duba A (1980) Effect of pressure and stress on water transport in intact and fractured gabbro and granite. J Geophys Res 85:7059-7071

Underwood EE (1970) Quantitative stereology. Addison-Wesley, Reading

Walsh JB (1965) The effect of cracks on the compressibility rock. J Geophys Res 70:381-389

Watanabe T, Higuchi A (2014) A new apparatus for measuring elastic wave velocity and electrical conductivity of fluid-saturated rocks at various confining and pore-fluid pressures. Geofluids 14:372-378

Watanabe T, Higuchi A (2015) Simultaneous measurements of elastic wave velocities and electrical conductivity in a brine-saturated granitic rock under confining pressures and their implication for interpretation of geophysical observations. Prog Earth Planet Sci. https://doi.org/10.1186/ s40645-015-0067-0

Wong TF, Fredrich JT, Gwanmesia GD (1989) Crack aperture statistics and pore space fractal geometry of Westerly granite and Rutland quartzite: implications for an elastic contact model of rock compressibility. J Geophys Res 94:10267-10278

\section{Publisher's Note}

Springer Nature remains neutral with regard to jurisdictional claims in published maps and institutional affiliations. 